\title{
INFLUENCE OF FLIGHT CONTROL LAW ON SPIN DYNAMICS OF AERODYNAMICALLY ASYMMETRIC AIRCRAFT
}

\author{
BiLAL MALIK \\ Avionics Department, Institute of Avionics and Aeronautics, Air University, Islamabad, Pakistan \\ e-mail: ahmedbilal786@yahoo.com \\ SuHAIL AKHTAR \\ Department of Avionics Engineering, CAE, NUST, Rislapur, Pakistan; e-mail: suhail@cae.nust.edu.pk \\ JAHANZEB MASOOD \\ Mechanical Engineering Department, Institute of Avionics and Aeronautics, Air University, Islamabad, Pakistan \\ e-mail: jehanzeb.masud@mail.au.edu.pk
}

\begin{abstract}
In this paper, we analyze the spin dynamics of an aerodynamically asymmetric aircraft in open-loop configuration and also evaluate the performance of gain scheduled flight control law in improving dynamic characteristics of aircraft spin. A look-up tables based aerodynamic model is developed from static, coning and oscillatory coning rotary balance wind tunnel test data. As a starting point, all possible steady spin modes are identified by solving the aircraft dynamic model comprising moment equations. The influence of high-alpha yawing moment asymmetry on predicted right and left spin modes is discussed. Six degree of freedom simulations of left and right flat spins are performed in open-loop and closed-loop configurations with the flight control law. Our studies reveal that large amplitude oscillations in the angle of attack and sideslip observed in the open-loop configuration are significantly damped by the control law. The control law reduces the recovery time of the left flat spin. However, the aircraft natural tendency to rotate rightwards due to yawing moment asymmetry at high angles of attack renders flight control law ineffective in aiding the recovery of the right flat spin.
\end{abstract}

Keywords: aerodynamic model, steady spin modes, unsteady effects, simulations

\section{Nomenclature}

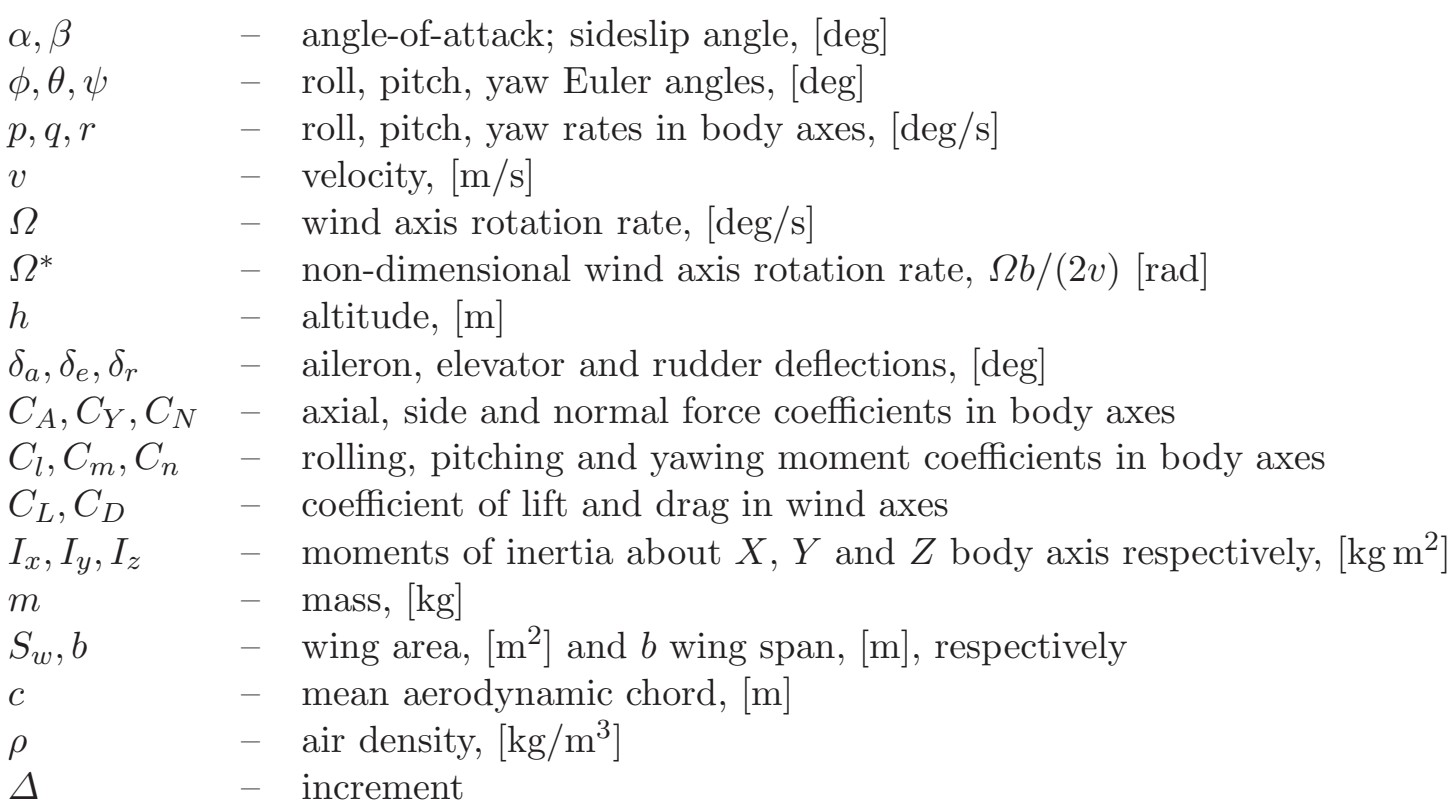




\section{Introduction}

Good high angle of attack characteristics are required for modern fighter aircraft to enhance maneuverability during actual combat and tactical training missions. While performing high-alpha maneuvers, fighter aircraft become susceptible to loss of controlled flight due to significant degradation in stability and control characteristics. Moreover, fighter configurations featuring long pointed nose and slender fuselage may also experience large asymmetric yawing moments at high angles of attack that result in loss of directional stability (Cobleigh, 1994). This phenomenon may cause yaw divergence and subsequent entry into spin, in the direction depending upon orientation of yawing moment asymmetry. Past statistics show that such inadvertent loss of control caused numerous stall/spin related accidents resulting in loss of aircraft/aircrew or both. As a result, prediction and analysis of post stall maneuvers using wind tunnel data and computer techniques in the early design/production stage of aircraft has received enormous attention from aeronautical engineering community.

A detailed account of evolution of aircraft spin and corresponding research to meet the challenges, since early years of aviation, was given by Abzug and Larrabee (2005). Aerodynamic models developed around the 1930's for studying aircraft spin were based on Bryan and Hartley (1911), which assumed that instantaneous values of aerodynamic coefficients depended only on instantaneous values of flight parameters and also that those coefficients varied linearly with those parameters. Such aerodynamic models, though suited for analyzing low angle of attack flights, proved inaccurate and inadequate for predicting and analyzing aircraft spin characteristics, because such high-alpha maneuvers involve aerodynamic loads that are highly nonlinear and show time lag with respect to flight parameters. Considerable efforts have, therefore, been put in by various countries in design and development of dynamic wind tunnel test facilities like rotary balance apparatus, forced oscillation motion rigs, oscillatory coning motion rigs, etc., that can emulate aircraft motion in post stall maneuvers (Bergmann, 2009; Jin et al., 2015; Zhang et al., 2015). Aerodynamic data from such dynamic wind tunnel test setups has been used effectively in identifying aircraft steady spin modes and simulation of post stall maneuvers (Bihrle, 1990; Murch and Foster, 2007; Khrabrov et al., 2013; Paul and Gopalarathnam, 2012).

Traditionally, a spin maneuver consists of three phases; incipient, fully developed spin and recovery phase. During incipient phase, aircraft transients from an aggravated stall to autorotation and flight path changes from horizontal to vertical. In the fully developed or steady spin phase, equilibrium is attained between inertia and aerodynamic forces and moments, and the aircraft descends downwards in a spiral trajectory about the vertical spin axis. The steady spin phase is extremely critical because if requisite anti-spin controls are not applied promptly, the spin may continue until the aircraft hits the ground. In the spin recovery phase, normally initiated by control inputs, the rotation rate decreases, aircraft nose attitude steepens, airspeed builds up and aircraft returns to the low-alpha flight regime. Bifurcation analysis and numerical continuation algorithms were used in the past to predict characteristics of steady spin such as flat or steep, stable or unstable, left or right, erect or inverted, steady or oscillatory, etc. (Paranjape and Ananthkrishnan, 2010). However, such methods present only a quasi-static state of the aircraft dynamics, and do not provide any details about transient motion (Raghavendra et al., 2005). Numerical continuation methods also require smooth functional representation of aerodynamic loads; as a result they are not suitable for look-up tables based aerodynamic models (Kolesnikov and Goman, 2012).

This paper addresses the problem of analyzing spin dynamics of a fighter aircraft cosponsored by China and Pakistan (Hewsom, 2005). As a starting point, all possible steady spin modes are identified by numerically solving the aircraft dynamic model comprising moment equations. Influence of high-alpha yawing moment asymmetry on identified left and right spin modes is illustrated with a steady spin modes location diagram. Flight parameters corresponding to the spin 
modes are used as initial conditions for six degree-of-freedom simulations of the aircraft spin. For this purpose, the flight dynamic model of the aircraft is developed in MATLAB/SIMULINK environment that can accurately simulate post-stall maneuvers. Six degree of freedom simulations of the aircraft spin are performed in open-loop configuration to study spin dynamic characteristics. Simulations in closed-loop configuration are also performed to evaluate the performance of the gain scheduled flight control law in improving aircraft spin dynamic characteristics and aiding spin recovery.

The past spin research focused on general aviation and fighter configurations that either did not experience aerodynamic asymmetries at high angles of attack or influence of such aerodynamic asymmetries on dynamic characteristics of spin are not addressed explicitly (Tischler and Barlow, 1981; Sibilski and Wróblewski, 2012). The work presented in this paper is, therefore, aimed at providing better physical insight into the effect of high-alpha yawing moment asymmetry on spin dynamics and recovery characteristics by performing computer simulations of left and right flat spins. Our results show that computer simulations can be effectively used in predicting and analyzing the key dynamic characteristics of aircraft spin like dynamic stability, rotation rate about spin axis, number of rotations before recovery, altitude loss during spin recovery, and contribution of the control law in aiding spin recovery, etc.

\section{Aircraft flight dynamics model}

The aircraft flight dynamical model is based on full six degree of freedom nonlinear equations of motion and utilizes the aerodynamic model stored in the form of look-up tables. The model also incorporates the actuator dynamics with range saturations and rate limitations, sensor dynamics and engine dynamics.

\subsection{Development of aerodynamic model}

A high fidelity aerodynamical model, in the form of look-up tables, is developed using experimental data from static, rotary balance coning motion and oscillatory coning motion tests. Broadly, each aerodynamic coefficient in the system of body axes can be represented as the sum of three components: the static aerodynamic component, increment due to steady rotations and increment due to unsteady aerodynamic effects

$$
C_{i}=C_{i, \text { static }}+\Delta C_{i, \text { steady }}+\Delta C_{i, \text { unsteady }}
$$

where $C_{i}=C_{A}, C_{Y}, C_{N}, C_{l}, C_{m}, C_{n}$.

The static component $C_{i, \text { static }}$ is measured from static wind tunnel tests for the angle of attack range $-45^{\circ}<\alpha<90^{\circ}$ and sideslip angles $-15^{\circ}<\beta<15^{\circ}$. The static aerodynamic component is modeled as

$$
\begin{aligned}
& C_{i, \text { static }}=C_{i}(\alpha, \beta, \delta=0)+\Delta C_{i}\left(\alpha, \delta_{a}\right)+\Delta C_{i}\left(\alpha, \delta_{e}\right)+\Delta C_{i}\left(\alpha, \delta_{r}\right) \\
& \quad+\Delta C_{i}\left(\alpha, \delta_{e}, \delta_{r}\right)+\Delta C_{i}\left(\alpha, \beta, \delta_{r}\right)
\end{aligned}
$$

The first term on the right-hand side of equation (2.2) represents the static effect for different angles of attack and sideslips, with all control surfaces set at the neutral position. The 2-nd to 4-th terms represent increments due to deflections of the aileron, elevator and rudder, respectively. The last two terms are aerodynamic increments due to rudder-horizontal tail and rudder-sideslip couplings. 
The steady aerodynamic component $\Delta C_{i, s t e a d y}$ is measured from rotary balance coning motion tests carried out on an aircraft model over the same range of aerodynamic angles as static tests and rotation rates between $-700^{\circ} / \mathrm{s}$ and $700^{\circ} / \mathrm{s}$. The steady aerodynamic component is modeled as

$$
\Delta C_{i, \text { steady }}=C_{i}\left(\alpha, \beta, \Omega, \delta_{a, e, r}=0\right)+\Delta C_{i}\left(\alpha, \Omega, \delta_{a}\right)+\Delta C_{i}\left(\alpha, \Omega, \delta_{e}\right)+\Delta C_{i}\left(\alpha, \Omega, \delta_{r}\right)
$$

The first term on the right hand side of equation (2.3) represents the rotational effects with aerodynamic controls at the neutral position; whereas the last three terms represent the aerodynamic increment due to the ailerons, elevator and rudder deflections, respectively. Each individual term in equations (2.2) and (2.3) represent an $n$-dimensional lookup table, where $n$ is the number of independent variables. Values of the coefficients are obtained by linear interpolation between the table nodes.

In coning motion dynamic wind tunnel tests, the aircraft model emulates a steady spin flight and, hence, the data from these tests is used to predict steady spin modes of the aircraft (Bihrle, 1990). During post stall maneuvers like oscillatory spins, incipient spins, spin recovery, etc., aerodynamic effects are highly nonlinear and time dependent (Bergmann, 2009). Accurate modeling of these unsteady aerodynamic effects is essential for correct simulations of the aircraft flight during such post stall maneuvers.

\subsection{Modeling of unsteady aerodynamic effects}

Generally, two approaches are followed to address the problem of modeling unsteady aerodynamic effects; computational fluid dynamics (CFD) (Luchtenburg et al., 2015; Ghoreyshi et al., 2014) and an experimental approach where the data from wind tunnel tests is used in conjunction with the system identification (SID) to obtain an adequate mathematical model from the data, such as a differential equation model (Abramov et al., 2004), neural network model (Ignatyev and Khrabrov, 2015), support vector machine (Wang et al., 2015), etc. Modeling of unsteady aerodynamic effects using CFD have shown very promising results, however its use in aircraft simulation applications remains limited because simultaneous solution of equations of fluid dynamics in conjunction with integration of aircraft equations of motion become extremely resource consuming. In the present work, a differential equation based model of the following form is used for the modeling of unsteady aerodynamic effects

$$
\tau_{i}(\alpha) \frac{d \Delta C_{i, u n s t e a d y}}{d t}+\Delta C_{i, \text { unsteady }}=k_{i}(\alpha) \dot{\alpha}
$$

The coefficient $k$ represents the instantaneous response, whereas the time constant $\tau$ corresponds to the time lag response. The values of $\tau$ and $k$ are computed from oscillatory coning motion test data using the least squares method. In Fig. 1, the model for the unsteady aerodynamic effect in the normal force coefficient is plotted with oscillatory coning data, whereas Fig. 2 shows the same plot for the pitching moment coefficient. The modeling is done for three different mean angles of attack $\alpha_{0}=35^{\circ}, 40^{\circ}, 50^{\circ}$. It is observed that the proposed model response in the two cases is reasonably close to the experimental data. The unsteady aerodynamic models are integrated with the overall aircraft dynamic model for simulation of flat oscillatory spins.

\subsection{High angle of attack aerodynamics}

Wind tunnel tests carried out on an aircraft model reveal two aerodynamic phenomena in the high angle of attack regime: strong aerodynamic asymmetry in the yawing moment and ineffectiveness of the rudder at high angles of attack. Variation of the static yawing moment coefficient $C_{n}$ with the angle of attack is illustrated in Fig. 3 for three rudder settings. 

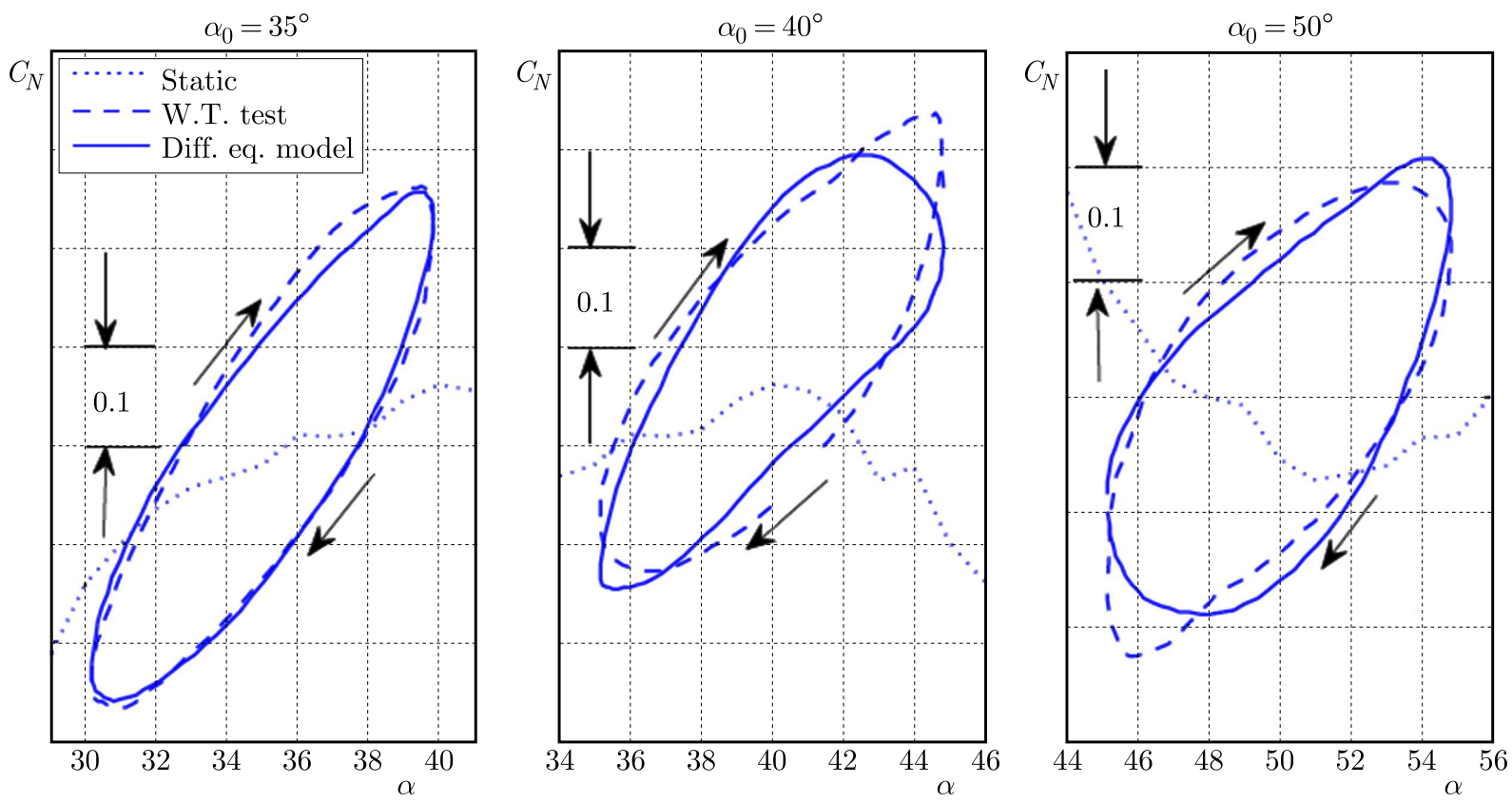

Fig. 1. Unsteady aerodynamic model response compared with test data: normal force $C_{N}$
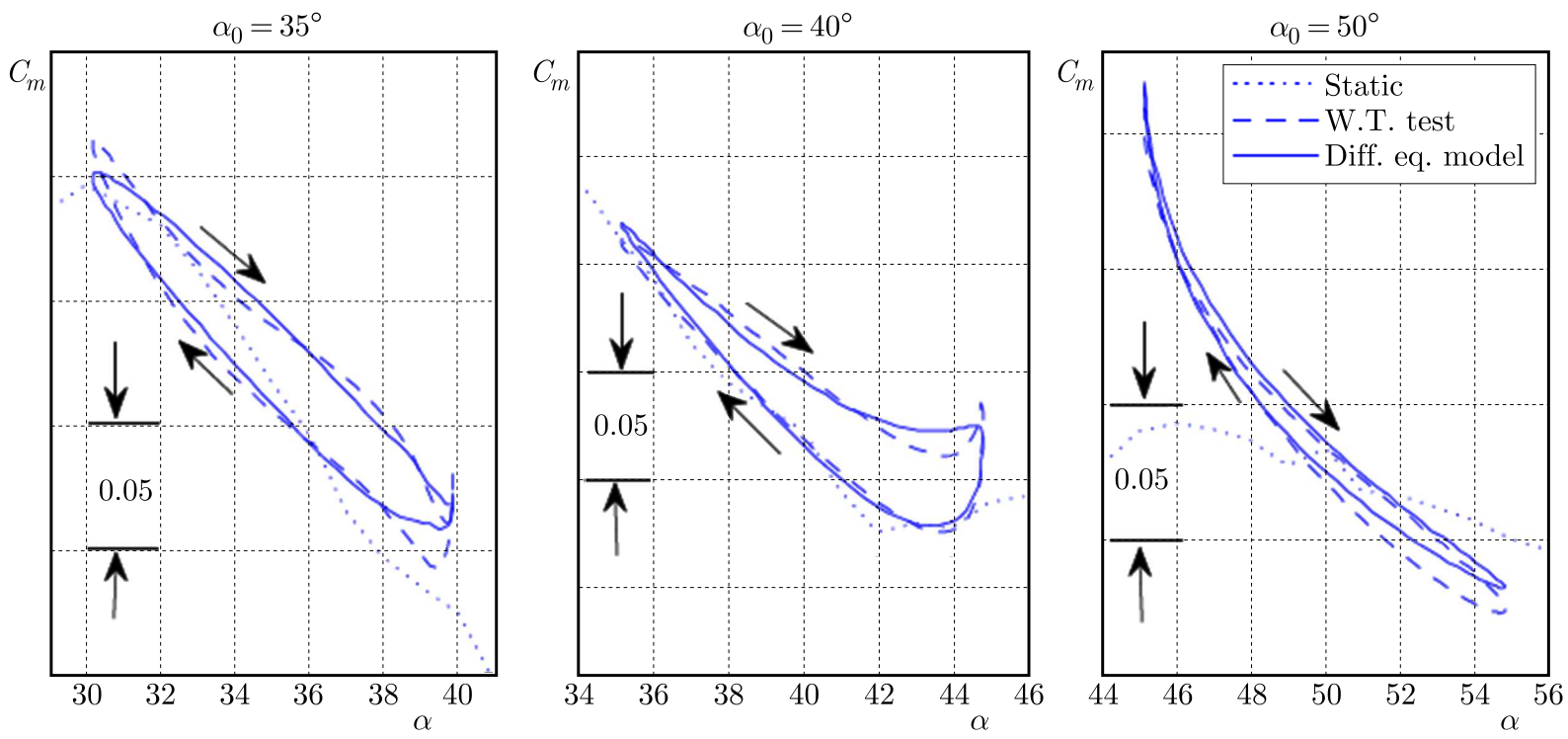

Fig. 2. Unsteady aerodynamic model response compared with test data: pitching moment $C_{m}$

As seen in Fig. 3, the yawing moment coefficient $C_{n}$ remains symmetric below $40^{\circ}$ angles of attack region, which is expected. However, as the angle of attack increases beyond $40^{\circ}$, strong asymmetry in the yawing moment is observed. Since the yawing moment coefficient $C_{n}$ is seen to remain positive in the region of asymmetry, the aircraft has inherent tendency to yaw towards right thus promoting departure and spin entry in the same direction. The exact reason of the observed high angle of attack yawing moment asymmetry is under investigation by conducting further wind tunnel tests and extended CFD simulations. Moreover, for angles of attack below $40^{\circ}$, the rudder is observed to be effective. As the angle of attack exceeds $40^{\circ}$, the rudder deflection does not produce a corresponding change in yawing moment, thus rendering the rudder ineffective at high angles of attack. This inefficiency of the rudder is due to influence of the wing and fuselage wake on the vertical tail surface. 


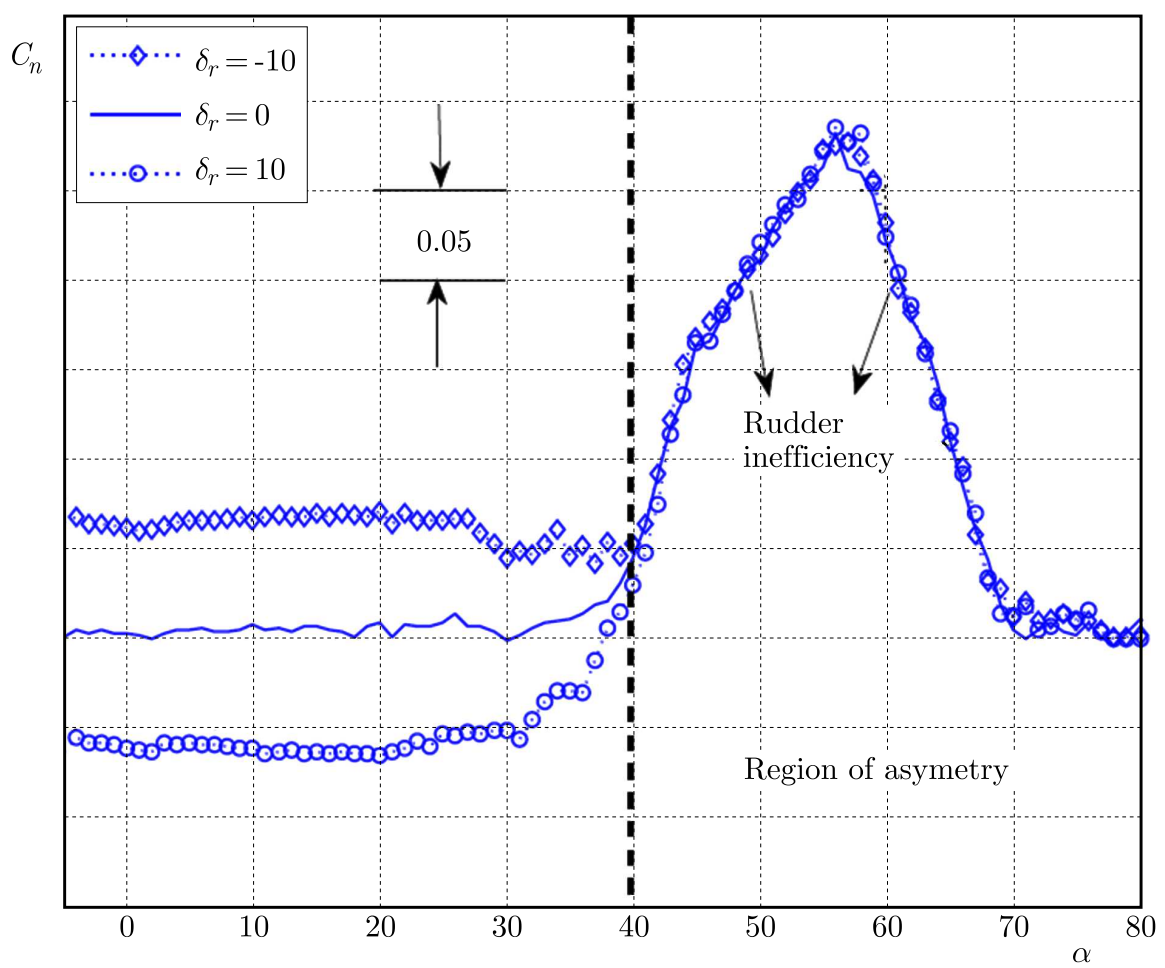

Fig. 3. Variation of yawing moment coefficient $C_{n}$ with angle of attack

\subsection{Control law architecture}

Flight control systems of modern fighter aircraft are designed to attain benefits like stabilization and control of a relatively unstable airframe, enhanced maneuverability at high angles of attack, automatic departure/spin prevention, enhanced safety and reduced pilot workload. This section presents high level description of the gain-scheduled flight control law selected for studying closed-loop spin dynamics. Conceptually, the control law can be divided into two main sections: longitudinal control law and lateral/directional control law. Functional block diagrams of longitudinal and lateral/directional control laws are illustrated in Figs. 4 and 5, respectively.

Each main section of the control law comprises of three main functional units: a command shaping module, feed-forward path and feedback path. The control law accepts pilot pitch, roll and yaw commands through longitudinal, lateral stick deflections and rudder pedals, respectively. These pilot inputs are fed to the command shaping modules where they are processed by a stick gradient, command limiter and command filter sub-modules to provide desired command augmentation properties. The outputs of the command shaping modules are then summed with the feedback signals to form command error signals $e p, e r$ and $e y$. The pitch error signal $e p$ is multiplied with the main gain of the longitudinal flight control path $K p$ to form the feedforward command which after passing through a proportional plus integral compensation filter is sent to the horizontal tail actuator. The roll error signal er after multiplication with the main gain of the roll control law $K r$ and passing through the proportional compensation filter is sent to the aileron actuator. Similarly, the yaw error signal ey is multiplied with the main gain of the yaw control law $K y$ which after passing through the proportional compensation filter is sent to the rudder actuator.

The dynamic feedback compensation module contains lead-lag filters and washout filters for achieving requisite gain and phase margins and improving the damping response, respectively. The notch filters in the feedback path attenuate the aircraft structural modes. The alpha-limiting 


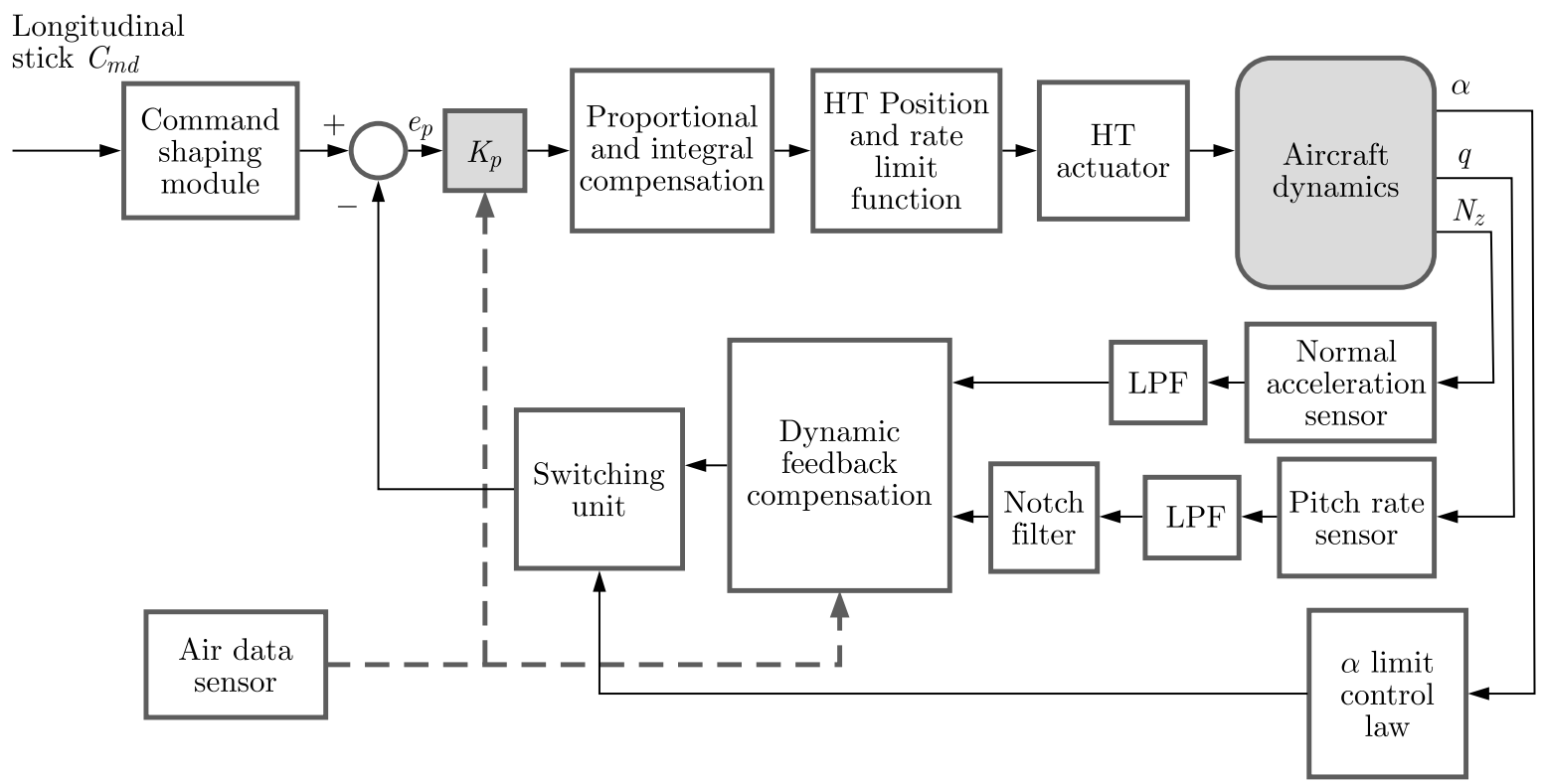

Fig. 4. Longitudinal control law: block diagram

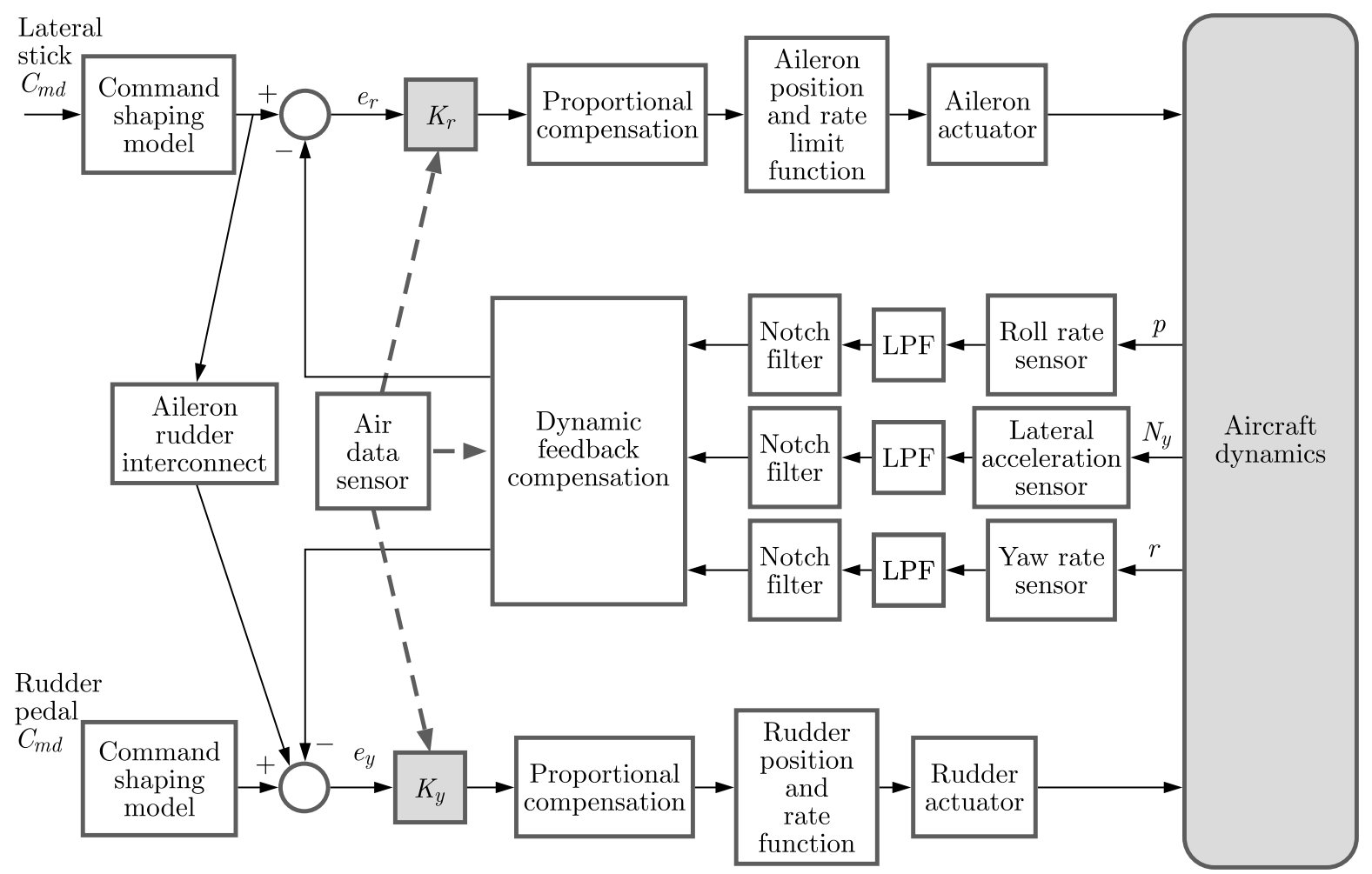

Fig. 5. Lateral-directional control law: block diagram

control law in the longitudinal feedback prevents aircraft excursions beyond the requisite angle of attack limits. The Aileron Rudder Interconnect (ARI) is provided to mitigate some of the adverse yaw effects encountered in fighter aircraft by aileron deflection, particularly at high angles of attack. 


\section{Identification of steady spin modes}

The first step in the prediction of aircraft spin characteristics is the identification of all possible steady spin modes and their sensitivity to aerodynamic control settings. The aircraft equations of motion in the steady spin phase were derived in detail by Pamadi (2004). In the steady spin phase, all accelerations along and about the aircraft body axes system are zero. Figure 6 illustrates forces acting on the aircraft in the steady spin. The resultant aerodynamic force acts normal to the wing chord line and the side-force is negligibly small. The resultant aerodynamic force is decomposed into two components: the lift $(L)$ acting in the horizontal direction and the drag $(D)$ in the vertical plane. The force equilibrium condition is satisfied when the drag balances aircraft weight $(W)$ and the lift balances the centrifugal force acting on the aircraft

$$
D=\frac{1}{2} \rho v^{2} S_{w} C_{D}=W \quad L=\frac{1}{2} \rho v^{2} S_{w} C_{L}=m r \Omega^{2}
$$

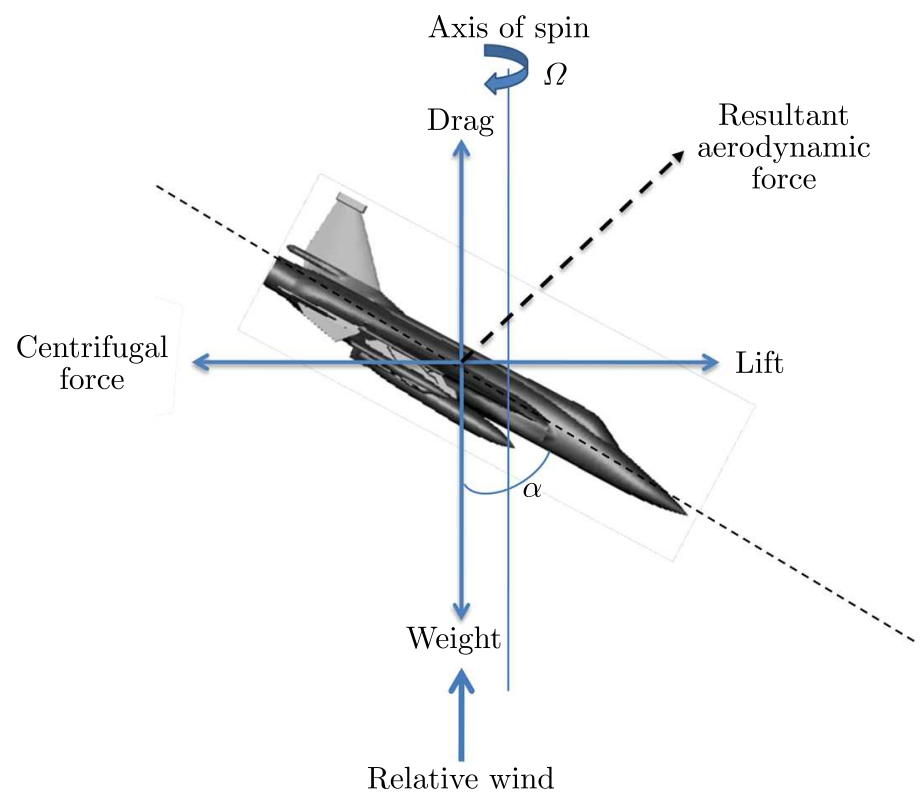

Fig. 6. Aircraft in steady spin

With the above stated flight conditions, the force equations are decoupled from the aircraft moment equations. Since force equilibrium can be attained at any attitude and spin rate by adjusting the spin radius and rate of descent, it is the balance of inertial and aerodynamic moment terms in the following moment equations that must be satisfied to ensure the steady spin condition

$$
\begin{aligned}
& \frac{4\left(I_{y}-I_{z}\right)}{\rho S_{w} b^{3}} \Omega^{* 2} \sin \alpha \sin (2 \beta)+C_{l}\left(\alpha, \beta, \Omega^{*}\right)=f_{1}(\mathbf{X}, \mathbf{U}) \\
& \frac{4\left(I_{z}-I_{x}\right)}{\rho S_{w} \bar{c} b^{2}} \Omega^{* 2} \sin (2 \alpha) \cos ^{2} \beta+C_{m}\left(\alpha, \beta, \Omega^{*}\right)=f_{2}(\mathbf{X}, \mathbf{U}) \\
& \frac{4\left(I_{x}-I_{y}\right)}{\rho S_{w} b^{3}} \Omega^{* 2} \cos \alpha \sin (2 \beta)+C_{n}\left(\alpha, \beta, \Omega^{*}\right)=f_{3}(\mathbf{X}, \mathbf{U})
\end{aligned}
$$

where $\mathbf{X}=\left[\alpha, \beta, \Omega^{*}\right]$ represents the state vector and $\mathbf{U}=\left[\delta_{a}, \delta_{e}, \delta_{r}, h, m\right]$ the control input. Equilibrium is reached when the inertial terms (first terms on the left hand side of equations (3.2)) balance the aerodynamic terms (second terms on the left-hand side of the same equations). Since the inertial as well as aerodynamic terms are functions of $\mathbf{X}$, the steady spin modes are 
computed by simultaneous solution of the moment equations for the unknown state vector $\mathbf{X}$ at a specific control input $\mathbf{U}$. Locations of the computed left and right spin modes in terms of the angle of attack, for the complete range of aileron deflections and for all elevator settings, are illustrated in Fig. 7. As seen, the right spin modes are significantly higher than the left spin modes. This correlates well with fact that high-alpha yawing moment asymmetry results in natural tendency of the aircraft to yaw toward right thus promoting departure and spin entry in the same direction.

Locations of the spin modes in Fig. 7 provide insight into their recoverability characteristics. As highlighted by Bihrle and Barnhart (1983), if the spin mode exists for pro-spin control settings and none for recovery or neutral control settings, the recovery is assisted by setting controls at positions where the spin mode does not exist. As seen in Figs. 7a and 7b, the left flat spin modes exist for elevator settings at $0^{\circ}$ and $-30^{\circ}$ and for the aileron deflection range $\delta_{a}<0^{\circ}$. No left flat spin modes exist for the elevator settings at $15^{\circ}$ (Fig. 7c). Hence, the recovery of left flat spins seems to be aided if the elevator is fully pitched down, i.e. $\delta_{e}=15^{\circ}$ and the aileron set to $\delta_{a} \geqslant 0^{\circ}$. However, for the right flat spins, which exist for all elevator settings and across the complete range of aileron deflections, the recovery through control surfaces input is seen to be problematic.
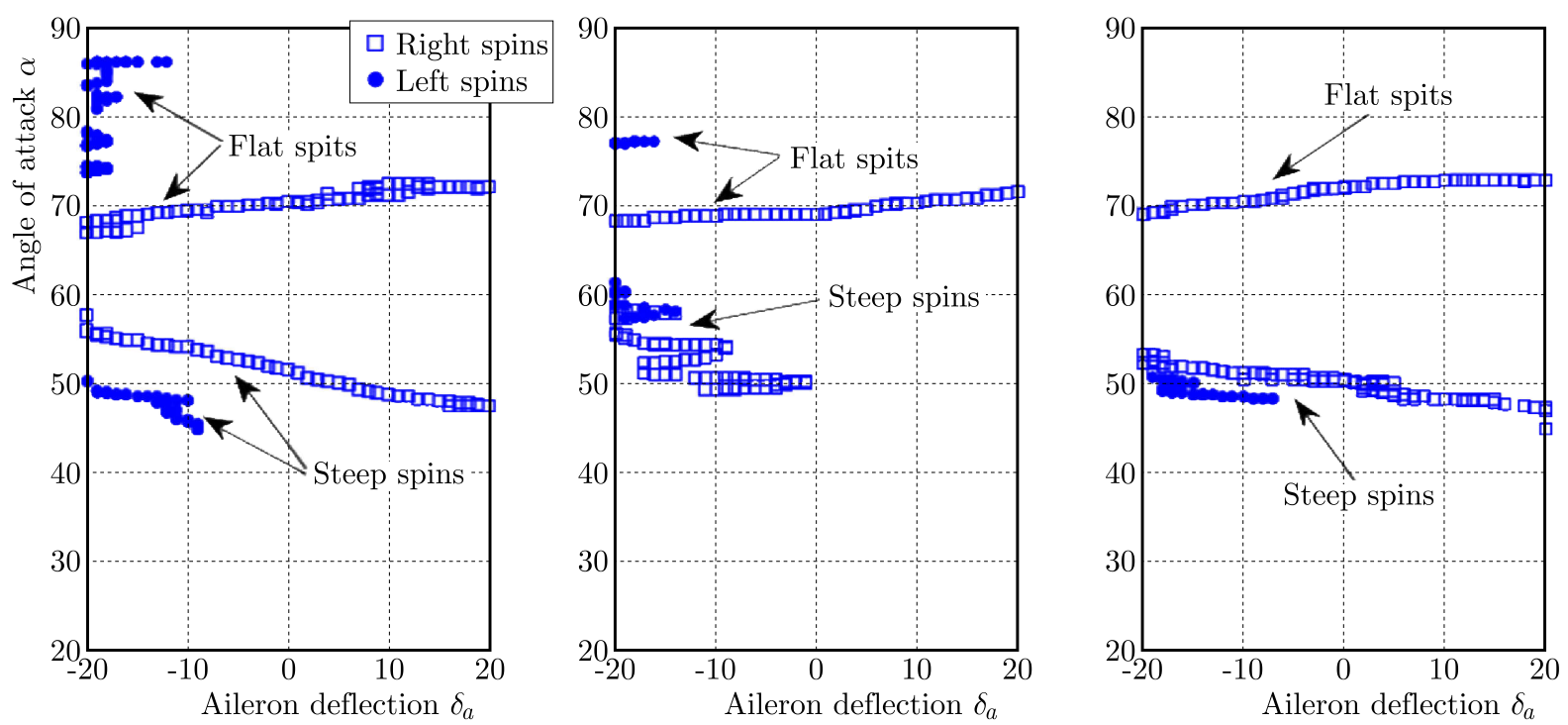

Fig. 7. Locations of steady spin modes for various elevator settings: (a) $\delta_{e}=0^{\circ}$, (b) $\delta_{e}=-30^{\circ}$, (c) $\delta_{e}=15^{\circ}$

\section{Time history simulations}

Time history simulations provide clear understanding on dynamic stability of identified spins, their oscillatory characteristics and effectiveness of flight control law in aiding the spin recovery. Simulations are carried out in MATLAB/SIMULINK environment, by integrating the full set of aircraft non-linear equations using the fourth-order Runge-Kutta routine. The force equations are taken in the aircraft wind axis and the moment equations are taken in the body axis system. Time history simulations are initialized with flight parameters corresponding to the aircraft steady spin and the results are demonstrated for two flat spins, left and right, listed in Table 1. The aircraft is said to be recovered from spin when the angle of attack reduces to $20^{\circ}$, which is safely below the stall region, and attain full effectiveness of control surfaces. 
Table 1. Right and left flat spins: initial conditions

\begin{tabular}{|l|c|c|c|c|c|c|c|c|c|c|c|c|c|c|}
\hline & \multicolumn{10}{|c|}{ Spin type } \\
\cline { 2 - 14 } & $\alpha$ & $\beta$ & $\Omega^{*}$ & $v$ & $\phi$ & $\theta$ & $\psi$ & $p$ & $q$ & $r$ & $z$ & $\delta_{a}$ & $\delta_{e}$ & $\delta_{r}$ \\
\hline \hline Right flat spin & 72.1 & -3.1 & 0.08 & 59.3 & 0 & -11.9 & 0 & 28.3 & -5.2 & 88.3 & 3000 & 10 & 0 & 0 \\
\hline Left flat spin & 78.2 & 2.8 & -0.11 & 58.8 & 0 & -17.8 & 0 & -25.3 & -5.8 & -120 & 3000 & -20 & 0 & 0 \\
\hline
\end{tabular}

\subsection{Dynamic characteristics of right flat spin}

Time histories of the right flat spin in closed-loop configuration (solid curves) are plotted in Fig. 8 and compared with spin simulations in open-loop configuration (dashed curves). The right flat spin is oscillatory in nature, since the angle of attack (Fig. 8d) and sideslip (Fig.8e) exhibit oscillations about the steady spin equilibrium point. Natural spin recovery is seen to occur because as the time passes, the aircraft descends downwards (Fig. 8f), airspeed increases (Fig. 8g), rotation rate about the spin axis decreases (Fig. 8j), angle of attack decreases well below the stalling angle and the aircraft enters the low alpha flight regime (Fig. 8d), where the aerodynamic controls become fully effective to attain low alpha steady flight conditions. Since the aircraft recovers naturally from the steady spin state, the right flat spin is unstable or divergent. It takes about 2.5 rotations for the aircraft to recover naturally from spin (Fig. $8 \mathrm{~h}$ ).

(a)

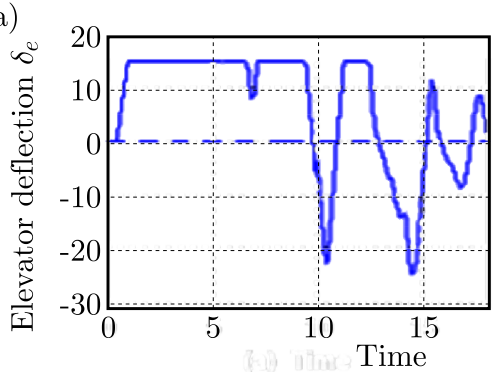

(d)

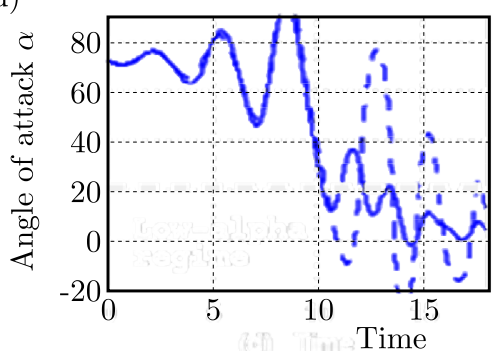

(g)

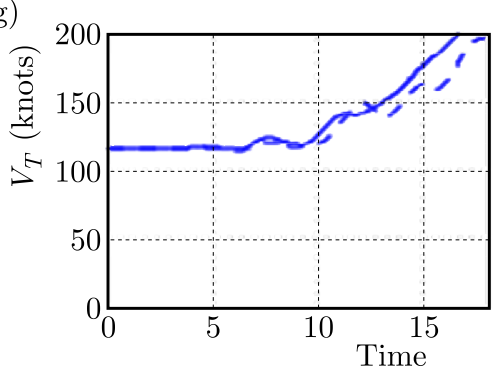

(b)

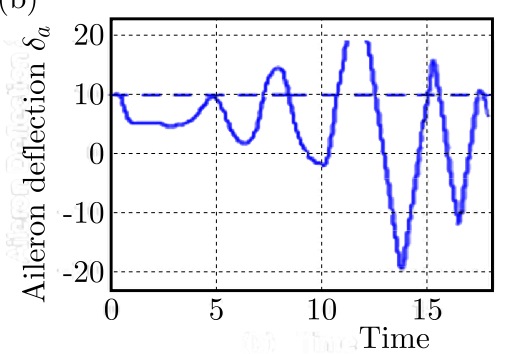

(e)

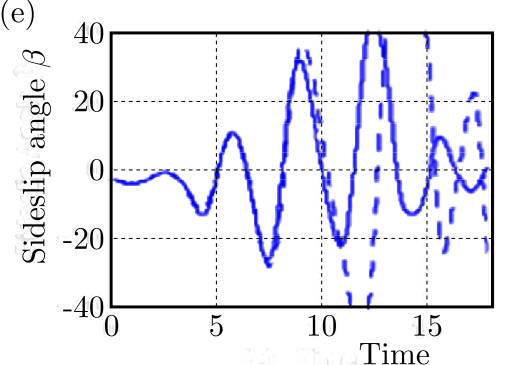

(h)

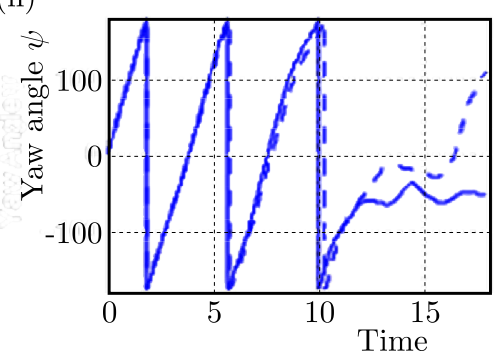

(c)

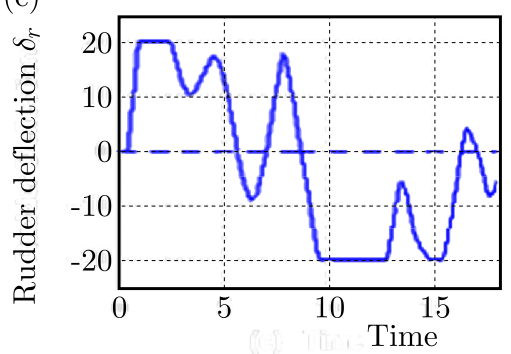

(f)
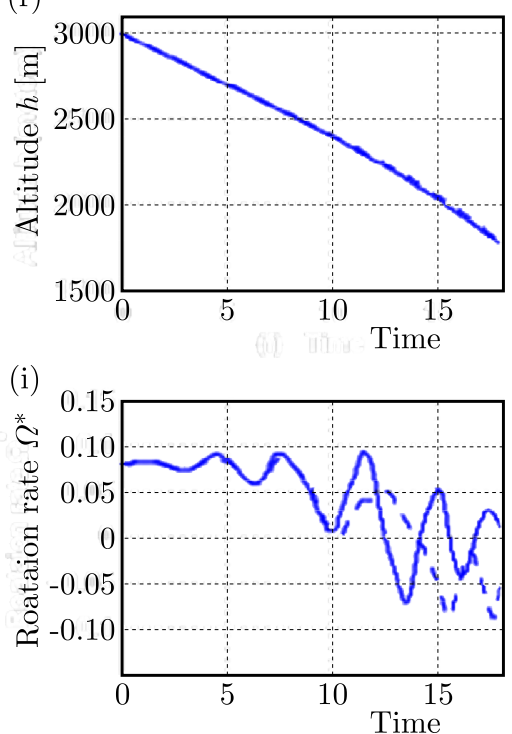

Fig. 8. Comparison of open loop (dashed) and closed loop (solid) time history simulations: right flat spin

It is seen from Fig. 8d that the gain scheduled flight control law does not reduce the recovery time of the right flat spin, since the aircraft enters the low-alpha flight regime at the same time in both cases, i.e. open-loop and closed loop configurations. The aircraft natural tendency to yaw rightwards due to high-alpha yawing moment asymmetry and existence of right steady 
spin modes across the entire aileron deflection range and elevator settings renders the control law ineffective in aiding the spin recovery. However, large amplitude oscillations in the angle of attack and sideslip (Fig. 8d and 8e), observed in the open loop spin dynamics, are significantly damped by the flight control law.

\subsection{Dynamic characteristics of left flat spin}

Time histories of the left flat spin in closed-loop configuration (solid curves) are plotted in Fig. 9 and compared with spin simulations in open-loop configuration (dashed curves). As observed from the time histories, the left flat spin is also oscillatory and divergent. As the spin gets flatter with the increasing angle of attack, the rotation rate about the spin axis increases and the recovery becomes difficult or may take a longer time (Pamadi, 2004). This phenomenon is observed in the case of the left flat spin, which is more flat $\left(\alpha=78.2^{\circ}\right)$ as compared to the right flat spin $\left(\alpha=72.1^{\circ}\right)$. The left flat spin in open-loop configuration is seen to have a high rotation rate and takes almost 17.5 seconds (Fig. 9d) and five turns (Fig. 9h) to transit from the steady spin flight phase to the low alpha-flight regime. It is observed that the gain scheduled flight control law is effective in reducing the recovery time of the left flat spin (Fig. 9d), and also damping of large amplitude oscillations in the angle of attack and sideslip observed in open-loop spin dynamics (Figs. 9d and 9e).

(a)

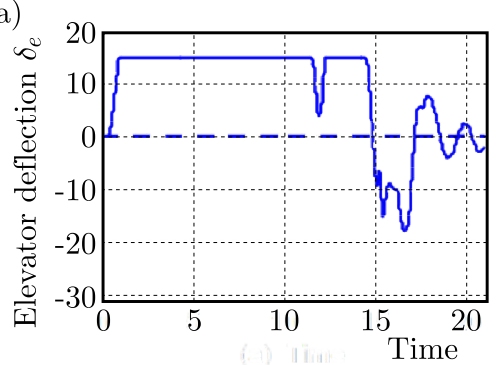

(d)

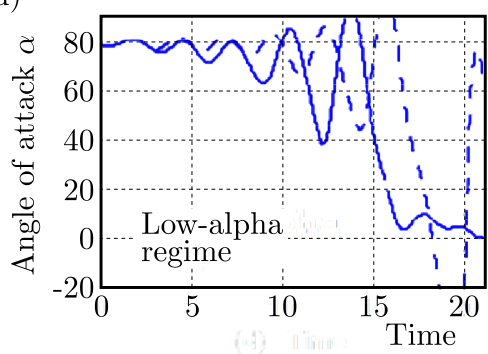

(g)

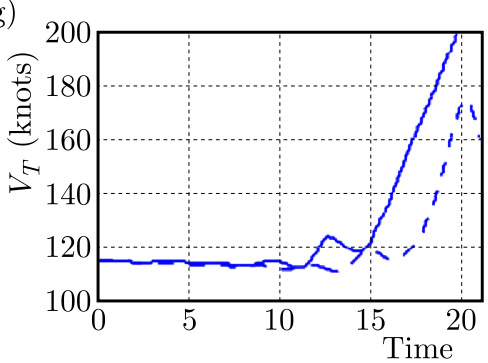

(b)

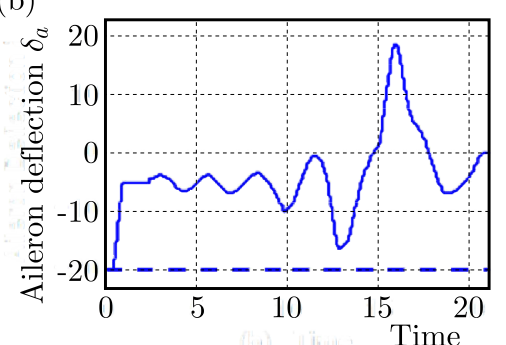

(e)

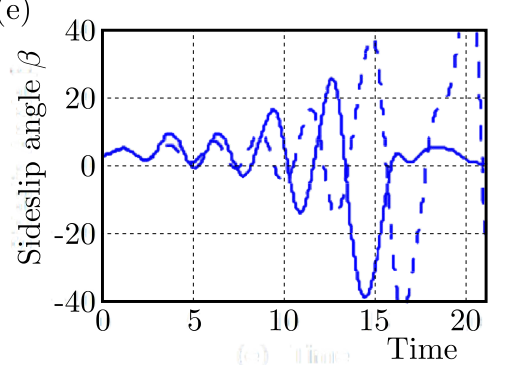

(h)

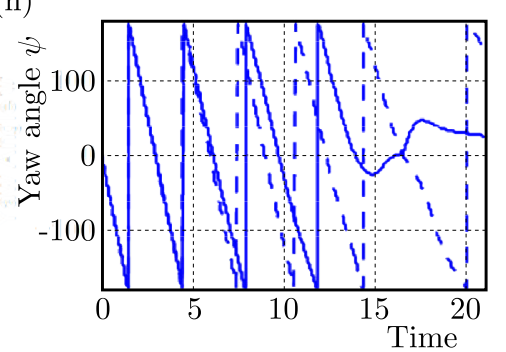

(c)

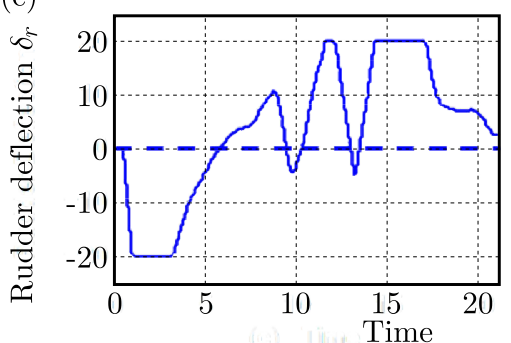

(f)
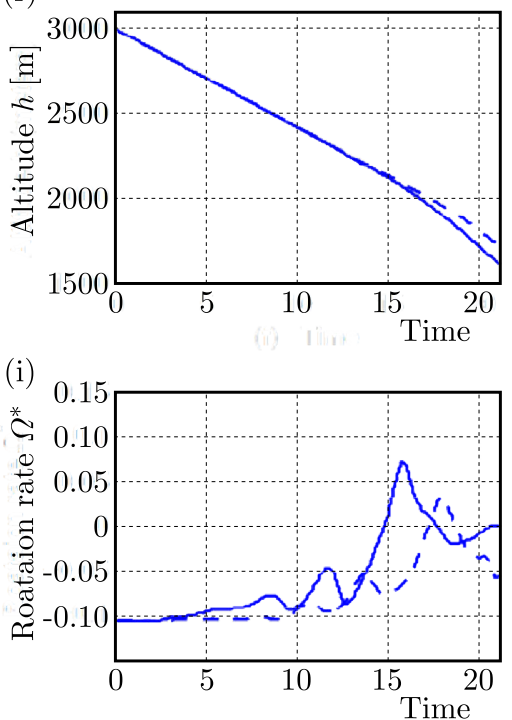

Fig. 9. Comparison of open loop (dashed) and closed loop (solid) time history simulations: left flat spin

The gain scheduled approach to designing of the flight controller has limitation since the controllers are designed only at equilibrium operating points within the prescribed flight envelope and therefore, may not cope with highly nonlinear aerodynamic effects encountered in the post stall flight regime. Moreover, problems may also be faced when scheduling with respect to rapidly changing state variables (Gill et al., 2015). To overcome such limitations, nonlinear control 
law designs such as dynamic inversion (Slotine and $\mathrm{Li}, 1991$ ) and various adaptive techniques (Crespo et al., 2012) may be tested with the aircraft flight simulation model for obtaining further improvements in dynamical characteristics of the aircraft spin.

\section{Conclusion}

The results presented in this paper demonstrate that computer simulations are an effective tool for prediction and analysis of dynamic characteristics of aircraft post-stall and spin maneuvers. For correct simulation of high alpha maneuvers, an accurate aerodynamic model which incorporates non-linear and unsteady aerodynamic effects encountered in such maneuvers is required. Whilst the dynamical system theory based approach facilitates understanding of asymptotic characteristics of the aircraft dynamics, time history simulations are vital to investigate dynamic effects in transitory flights such as dynamic stability of developed spins, ease or difficulty with which aircraft enters or recovers from a spin etc.

The spin dynamics of fighter configuration with high-alpha yawing moment asymmetry is analyzed in open loop and closed loop configurations, and the performance of the gain scheduled flight control law in improving recovery and oscillatory characteristics of fully developed spins is evaluated. The aircraft natural tendency to rotate towards right due to high alpha yawing moment asymmetry has strong influence on the predicted spin modes. The right flat spins are found to exist across the entire aileron deflections and for all elevator settings. The right and left flat spins are oscillatory and unstable. The left spin being more flat than the right one, takes longer to recover and has a higher rotation rate. The control law is effective in reducing the recovery time for left spins; however fails to do the same for right flat spins as they exist across the full range of ailerons deflections and all elevator settings. Moreover, the control law effectively damps large amplitude oscillations observed in the left and right flat spins in open loop configuration.

\section{References}

1. Abramov N., Goman M., Khrabrov A.N., 2004, Aircraft dynamics at high incidence flight with account of unsteady aerodynamic effects, AIAA Atmospheric Flight Mechanics Conference and Exhibit, Rhode Island

2. Abzug M.J., Larrabee E.E., 2005, Airplane Stability and Control: a History of the Technologies that Made Aviation Possible, Cambridge University Press, England

3. Bergmann A., 2009, Modern wind tunnel techniques for unsteady testing-development of dynamic test rigs, [In:] Hermann Schlichting - 100 Years, R. Radespiel, C.-C. Rossow, B.W. Brinkmann (Edit.), Springer Berlin Heidelberg, 59-77

4. Bihrle W., 1990, Use of rotary balance data in the prediction of aircraft dynamics, AGARD Advisory Report, No. 265, 188-208

5. Bihrle W., Barnhart B., 1983, Spin prediction techniques, Journal of Aircraft, 2, 97-101

6. Bryan, Hartley G., 1911, Stability in Aviation: An Introduction to Dynamical Stability as Applied to the Motions of Aeroplanes, Macmillan and Co. Limited

7. Cobleigh B.R., 1994, High angle of attack yawing moment asymmetry of the X-31 aircraft from flight test, NASA Contractor Report 186030, 1-36

8. Crespo L.G., Matsutani M., Annaswamy A.M., 2012, Design of an adaptive controller for a remotely operated air vehicle, Journal of Guidance, Control, and Dynamics, 2, 406-422 
9. Ghoreyshi M., Jirasek A., Cummings R.M., 2014, Reduced order unsteady aerodynamic modeling for stability and control analysis using computational fluid dynamics, Progress in Aerospace Sciences, 71, 167-217

10. Gill S.J., Lowenberg M.H., Neild S.A., Crespo L.G., Krauskopf B., Puyou G., 2015, Nonlinear dynamics of aircraft controller characteristics outside the standard flight envelope, Journal of Guidance, Control, and Dynamics, 12, 2301-2308

11. Hewsom R., 2005, Sino-Pakistani fighter improved, Jane's Defense Weekly, 99-100

12. Ignatyev D.I., Khrabrov A.N., 2015, Neural network modeling of unsteady aerodynamic characteristics at high angles of attack, Aerospace Science and Technology, 41, 106-115

13. Jin L., Nong C., Yuhui S., Jing H., Ke X., 2015, New dynamic stability rig for tri-sonic wind-tunnel, Procedia Engineering, 99, 1591-1596

14. Khrabrov A., Sidoryuk M., Goman M., 2013, Aerodynamic model development and simulation of airliner spin for upset recovery, Progress in Flight Physics, 5, 621-636

15. Kolesnikov E., Goman M., 2012, Analysis of aircraft nonlinear dynamics using non-gradient based numerical methods and attainable equilibrium sets, AIAA Atmospheric Flight Mechanics Conference, Minnesota

16. Luchtenburg D.M., Rowley C.W., Lohry M.W., Martinelli L., Stengel R.F., 2015, Unsteady high-angle-of-attack aerodynamic models of a generic jet transport, Journal of Aircraft, 3, 890-895

17. Murch A.M., Foster J.V., 2007, Recent NASA research on aerodynamic modeling of post-stall and spin dynamics of large transport airplanes, 45th AIAA Aerospace Sciences Meeting and Exhibit, Nevada

18. Pamadi B.N., 2004, Performance, Stability, Dynamics, and Control of Airplanes, AIAA Education Series, USA

19. Paranjape A.A., Ananthkrishnan N., 2010, Analytical criterion for aircraft spin susceptibility, Journal of Aircraft, 5, 1804-1807

20. Paul R., Gopalarathnam A., 2012, Simulation of flight dynamics with an improved post-stall aerodynamics model, AIAA Atmospheric Flight Mechanics Conference, Minnesota

21. Raghavendra P.K., Sahai T., Kumar P.A., Chauhan M., Ananthkrishnan N., 2005, Aircraft spin recovery, with and without thrust vectoring, using nonlinear dynamic inversion, Journal of Aircraft, 6, 1492-1503

22. Sibilski K., Wróblewski W., 2012, Prediction of aircraft spin characteristics by continuation and bifurcation methods, AIAA Atmospheric Flight Mechanics Conference, Minnesota

23. Slotine J.J., Li W., 1991, Applied Nonlinear Control, Prentice Hall, Engle-wood Cliffs, New Jersey

24. Tischler M.B., Barlow J.B., 1981, Determination of the spin and recovery characteristics of a general aviation design, Journal of Aircraft, 4, 238-244

25. WANG Q., QIAN W., HE K., 2015, Unsteady aerodynamic modeling at high angles of attack using support vector machines, Chinese Journal of Aeronautics, 3, 659-668

26. Zhang J., Tang Y.K., Sun H.S., Liu Z.T., 2015, Dynamic test experiment system of single degree of freedom of $\emptyset 3.2 \mathrm{~m}$ wind tunnel, Proceedings of the Institution of Mechanical Engineers, Part G: Journal of Aerospace Engineering, 7, 1300-1309 